\title{
In memoriam of Prof. Mario Andreoli
}

Pubblicato online: 17 febbraio 2017

C) Springer International Publishing AG 2017

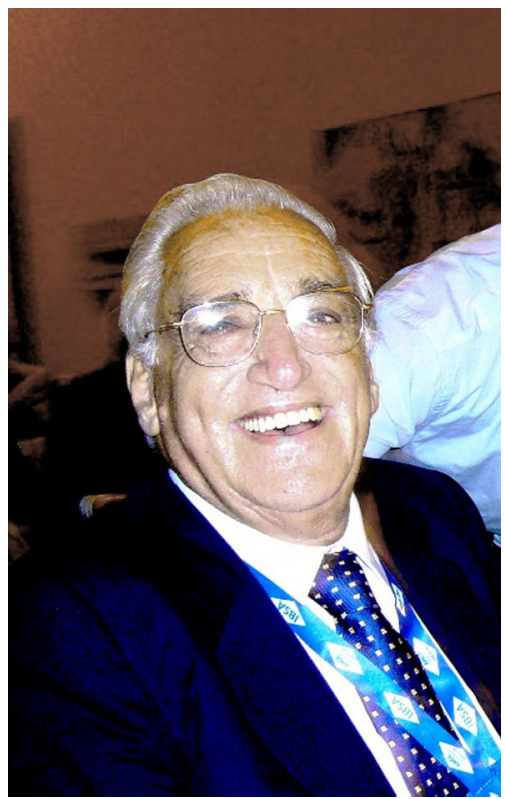

Il Prof Mario Andreoli, nato a San Lucido (CS) il 21/8/1929 è mancato il 9 Maggio 2016 a Roma. Allievo del Prof. Cassano prima presso la Clinica Medica dell'Università di Pisa e poi dal 1956 presso la Patologia Medica e quindi della II Clinica Medica dell'Università di Roma.

Primo endocrinologo italiano a recarsi presso la Clinical Endocrinology Branch, N.I.A.M.D., dei National Institutes of Health, negli anni 1962-1963.

$\mathrm{Al}$ suo rientro a Roma costituì un prestigioso gruppo di ricerca di studiosi di fisiopatologia tiroidea.

Segretario della Fifth International Thyroid Conference 24-27 May 1965.
Segretario della European Thyroid Association dal 1967 al 1971.

Fondatore e Direttore del Centro di Fisiopatologia tiroidea del CNR dal 1973 al 1980.

Professore incaricato di Endocrinologia prima presso l'Università di Sassari e poi Ordinario alla Università di Roma La Sapienza dal 1980.

Professore Ordinario di Endocrinologia all'Università "La Sapienza" di Roma dal 1980 al 2001.

Professore Emerito dell'Università di Roma La Sapienza dal 2002.

Direttore della II Scuola di Specializzazione in Endocrinologia dell'Università di Roma La Sapienza.

Presidente della Società Italiana di Endocrinologia 19921994. Il prof. Andreoli ha contribuito in maniera determinante allo sviluppo e crescita dell'Endocrinologia e in particolare della Tireologia in Italia. La sua inesauribile passione e amichevole attitudine sarà ricordata da tutti coloro che lo conobbero. 